\title{
Evaluation of a new enzyme-linked immunosorbent assay test for rotavirus antigen in faeces
}

\author{
GM BEARDS, AS BRYDEN
}

From the Regional Virus Laboratory, East Birmingham Hospital, Birmingham B9 5ST, and the Department of Virology, Preston Infirmary, Deepdale Road, Preston, Lancashire

SUMMARY A new commercial test for the diagnosis of rotavirus gastroenteritis was assessed. W'ith some modifications it compared favourably with electron microscopy and immunofluorescence.

Most laboratories are restricted to electron microscopy for the rapid diagnosis of rotavirus gastroenteritis. Other tests are available, but are dependent on the availability of specific antisera which are not easily obtained. These include counter-immune electrophoresis ${ }^{1}$ and complement fixation, ${ }^{2}$ both of which are relatively insensitive; immunofluorescence in cell culture (IF) ${ }^{3}$ which is comparable with electron microscopy; and the "third generation" tests: radioimmunoassay (RIA), ${ }^{4}$ and enzymelinked immunosorbent assay (ELISA). ${ }^{56}$

There is an urgent need for an inexpensive, simple, and rapid diagnostic test of high sensitivity for rotavirus gastroenteritis, particularly in Third World countries where infantile gastroenteritis is incriminated in millions of deaths each year.?

A problem with third generation tests such as ELISA is the antigenic and morphological variation

Accepted for publication 23 March 1981 associated with the rotavirus particle (GMB, to be published). In electron miscroscopic preparations of rotaviruses two distinct morphological forms are seen; complete or "smooth" particles, $65 \mathrm{~nm}$ in diameter, and incomplete or "rough" particles, 50-54 $\mathrm{nm}$ in diameter (Fig. 1). ${ }^{8}$ Incomplete particles are formed from complete ones by the loss of the outer capsid layer, or as a result of incomplete virus synthesis, and this conversion may be easily effected in vitro by treatment with a chelating agent-for example, ethylenediaminotetraacetate (EDTA). ${ }^{9}$ This treatment also results in loss of infectivity ${ }^{10}$ (ASB? unpublished).

Group-specific antigens are found on the surface of incomplete particles, ${ }^{11}$ whereas type-specific antigens are situated on the surface of complete particles. ${ }^{12}$ These type-specific antigens give rise to serotype variations between human strains of rotavirus which were first detected by Zissis and Lambert, ${ }^{13}$ and Thouless and coworkers, ${ }^{14}$ by

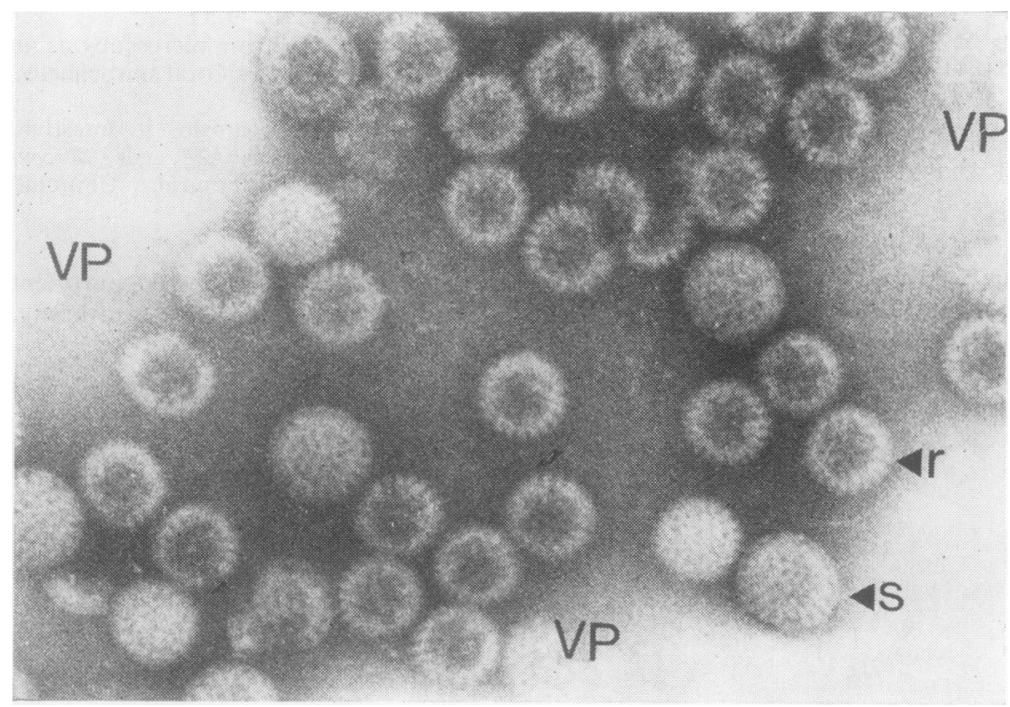

Fig. 1 Rotavirus particles in faeces. Both smooth $(s)$ and rough ( $r$ ) morphology is shown, along with some free viral protein (VP). Ammonium molybdate negative stain $\times 150000$. 
complement fixation and serum neutralisation respectively. Later Yolken ${ }^{15}$ confirmed these findings by an ELISA technique. At least four distinct serotypes of human rotavirus have now been described ${ }^{16}$ (and Thouless, to be published). Both complete and incomplete particles are present in faeces in variable proportions. $^{8}$

To be of value, any rapid serological diagnostic test should be based on the group-specific antigen otherwise false-negative results might occur, when complete particles of a different serotype are predominant in the sample being tested.

Recently Abbott Laboratories have introduced a commercial ELISA kit (Rotazyme) for the diagnosis of rotaviral gastroenteritis. We have compared this system with immunofluorescence and electron microscopy and tested its sensitivity against morphologically and antigenically different rotavirus isolates. Minor modifications to the test system are suggested which increase its sensitivity.

\section{Material and methods}

An examination was made on 171 samples of faeces from children suffering from gastroenteritis. An approximately $10 \%$ emulsion was prepared in phosphate-buffered saline (PBS) $\mathrm{pH} 7 \cdot 2$ containing suitable antibiotics and clarified by low speed centrifugation. Faeces from four calves, intestinal contents from one foal and intestines from three litters of infant mice, all of which had been infected with rotavirus, were similarly prepared and examined. A number of samples were received from abroad.

A sample of cell culture adapted bovine rotavirus and human isolates of different serotypes were also tested.

\section{IMMUNOFLUORESCENCE}

This was carried out as described by Bryden et al. ${ }^{3}$ LLC-MK $\mathbf{M}_{2}$ cells were again used but infection was demonstrated by a hyperimmune serum raised in a rabbit against human rotavirus and stained with FITC conjugated antiserum to rabbit IgG raised in sheep (Burroughs-Wellcome).

\section{ELECTRON MICROSCOPY}

This was carried out as described by Flewett et al., ${ }^{8}$ or faecal extracts in PBS were clarified by low speed centrifugation and concentrated by ammonium sulphate precipitation, absorbed on to carbon formvar-coated grids, and negatively stained with potassium phosphotungstate $\mathrm{pH} \mathbf{7 \cdot 0}$, as described by Caul et al. ${ }^{17}$

ROTAZYME

This was performed as suggested by the manufacturers except that $10 \%$ faecal extracts as described above, and not 1:3 faecal extracts in PBS, were used. Briefly, polystyrene beads coated with an antiserum prepared in guinea pigs to simian rotavirus (SA11) were exposed to $200 \mu \mathrm{l}$ of faecal extract for two to three hours at $45^{\circ} \mathrm{C}$. These were washed and then exposed to $200 \mu \mathrm{l}$ of rabbit antirotavirus serum conjugated to horseradish peroxidase for one hour at $45^{\circ} \mathrm{C}$. After incubation and washing, the beads were transferred to fresh tubes and $200 \mu \mathrm{l}$ of substrate, OPD (O-ethylenediamine-2 $\mathrm{HCl}$ ) in citrate phosphate buffer added. They were held for $15 \mathrm{~min}$ at room temperature in the dark. The reactions were stopped by addition of $1 \mathrm{ml} 1 \mathrm{~N} \mathrm{HCl}$ and read either visually or by measurement of absorbance at $492 \mathrm{~nm}$ (with a cut off value of 0.05 ). The kit incorporates a suspension of SA11 which was used as a positive control. To investigate the reproducibility of the Rotazyme test, seven samples were retested at least once. Comparative titrations in the presence and absence of EDTA were performed on two samples by diluting the extracts in PBS with or without 0.025 M EDTA (giving a final concentration of $0.005 \mathrm{M}$ ), incubating for one hour at $37^{\circ} \mathrm{C}$ and then tested by Rotazyme.

\section{Results}

The results of the 171 samples from human infants tested by Rotazyme, electron microscopy and immunofluorescence are given in Table 1. No conclusions about the relative virtues of immunofluorescence and electron microscopy should be drawn from these results as specimens giving discrepant results with these tests were deliberately introduced into the study.

The results of comparative titrations are illustrated in Figs. 2 and 3. In some samples a prozone effect was observed up to a $1 / 5$ dilution of the test

Table 1 Results of 171 specimens from infants tested for the presence of rotavirus, by Rotazyme, electron microscopy and immunofluorescence

\begin{tabular}{llllll}
\hline No tested & $\begin{array}{l}\text { Tested positive by at } \\
\text { least one test }\end{array}$ & Rotazyme + & EM/FA & EM + & FA + \\
\hline 171 & 69 & 69 & 65 & 55 & 52 \\
\hline
\end{tabular}

Four were positive by Rotazyme only, confirmed by our independent ELISA test.

Includes one EM positive which was positive by Rotazyme only after EDTA was included. 


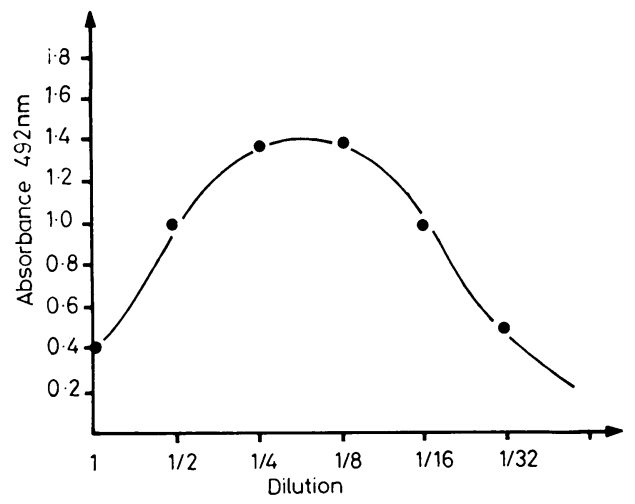

Fig. 2 Titration of antigen in faeces by the Rotazyme test. A prozone effect is demonstrated with this particular sample. Optimum results were obtained when the $10 \%$ faecal extract was diluted $1 / 4$ to $1 / 8$ in PBS.

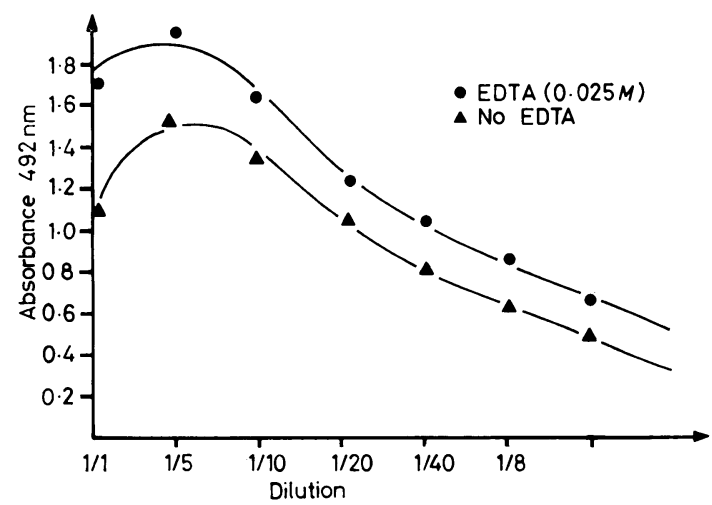

Fig. 3 Titration of one sample in the presence and absence of 0.025 M EDTA in PBS. Samples were diluted in the buffer containing EDTA and incubated at $37^{\circ} \mathrm{C}$ before testing.

extract. Five samples from Vellore in India which were positive by electron microscopy and immunofluorescence were only positive by the ELISA test when the prozone effect was reduced by dilution (Table 2). The results of further dilutions of other test samples were variable however, and many did not show an increase in absorbance when diluted.

The incorporation of EDTA $(0.025 M)$ was found to be beneficial. Five specimens gave higher absorbance values (mean absorbance difference $+0 \cdot 12$ ) but three did not show any significant difference. One specimen which was positive by EM and IF would have been recorded as ELISA-negative if EDTA had not been added. The differences in titrations are shown in Fig. 3. By removing the outer coat polypeptides from the virus particles and exposing the group antigens, EDTA has enhanced
Table 2 Results from Vellore samples tested by Rotazyme and EM

\begin{tabular}{llll}
\hline Sample & $\begin{array}{c}\text { At } 492 \mathrm{~nm} \\
\text { OD NEAT }\end{array}$ & OD $1 / 5$ & EM \\
\hline T3 & 0.029 & 0.139 & \\
D741 & 0.044 & 0.217 & \\
D696 & 0.020 & 0.064 & \\
P020 & 0.043 & 0.143 & $\vdots$ \\
P003 & 0.037 & 0.075 & - \\
T34 & 0.030 & 0.036 & - \\
\hline
\end{tabular}

Tested in parallel. Cut off value 0.050 .

Vellore samples kindly provided by Professor M Mathan.

the sensitivity of the test, suggesting that the Rotazyme antibody is directed mainly at internal polypeptides.

Specimens containing rotavirus from mice, foals, calves (including a tissue culture-adapted strain) and human isolates of different serotypes were all positive by Rotazyme.

The results of the reproducibility study are shown in Table 3. The average variation in absorbance was $0 \cdot 179$.

Table 3 Results of re-testing by Rotazyme of seven samples

\begin{tabular}{ll}
\hline Specimen & Results $($ absorbance at $492 \mathrm{~nm})$ \\
\hline 1 & $0 \cdot 1000 \cdot 100$ \\
2 & $0 \cdot 3500.6000 .6500 .550$ \\
3 & 0.4000 .3300 .420 \\
4 & 1.8001 .900 \\
5 & $0 \cdot 2500.220$ \\
6 & 0.3580 .6240 .4770 .5600 .5030 .6220 .443 \\
7 & 1.0951 .1651 .590 \\
\hline
\end{tabular}

Mean variation from first testing $=0 \cdot 179$.

\section{Discussion}

Rotazyme is obviously based on group-specificity since the incorporation of EDTA which exposed the group antigen(s) seems to enhance its sensitivity. In samples containing mostly incomplete particles the incorporation of EDTA will make little difference to the readings obtained but with samples containing a high percentage of complete particles the omission of EDTA might give rise to falsely negative or only weakly positive results. When comparing the titres of various extracts, EDTA should be included to ensure accuracy.

A spectrophotometer was found useful for this study, but for qualitative work and routine diagnosis we do not regard it as essential, as there was little difficulty in reading results visually following the manufacturer's instructions.

We cannot explain the prozone effect obtained with some samples. This phenomenon has been 
observed with immunofluorescence and an ELISA system established by ourselves. It is recommended that specimens positive by other tests but negative by Rotazyme are repeated after a dilution of $1 / 5$ in PBS.

On the whole the kit performed well, as it is both specific and simple to perform. We did not like the manufacturer's instruction to test specimens at $1 / 3$ dilution in PBS, as this material was inconvenient to handle. Most laboratories routinely make $10 \%$ wt/vol extracts in PBS clarified by a low speed centrifugation which can then be used for other tests as well. No problems were encountered using this type of extract.

The reproducibility was good. The variation is great enough to affect weak positive results, possibly giving rise to falsely negative readings or vice versa, but such borderline samples would probably have to be confirmed by a separate test, as a matter of routine.

The fact that the test detects rotaviruses from different species suggests that it may also be useful to veterinary workers, since death of livestock owing to gastrointestinal infections is a major economic problem in many countries. ${ }^{18}$

For a third generation test, however, the sensitivity is relatively low, and it is possible that the test has potential for greater sensitivity. One possible explanation is that some commercial firms may be reluctant to introduce a new test that gives positive results that are difficult to confirm by existing methods. This is a well known problem with techniques of high sensitivity such as ELISA. Our knowledge of disease-free excretion is limited however, and it might be shown that greater sensitivity is not desirable. But until the epidemiological significance of subclinical cases is firmly established there is a need for tests of high sensitivity.

Another disadvantage of the Rotazyme kit is the cost (at the moment it costs over $£ 1.00$ per test). Many laboratories would find it more economical to develop their own ELISA test. The greatest need for such a test as the Rotazyme lies with the Third World, but at present the cost involved seems prohibitive.

The authors wish to thank Mr AS Jobson and his colleagues in Abbott Laboratories Ltd, for the generous gift of the kits we have evaluated; Drs ME Thouless and TH Flewett for advice and incouragement; Drs Elizabeth Boxall and Susan Skidmore for critical help with the manuscript and Catherine Nichols for typing it. We are especially indebted to Miss Anne Brennan, Mrs Cheryl Hall and Mrs Jane Shirley for their help with the electron microscopy and immunofluorescence.
GMB is supported by a grant from the Medical Research Council.

\section{References}

1 Tufvesson B, Johnson T. Immunoelectro-osmophoresis for detection of reo-like virus: methodology and comparison with electron microscopy. Acta Pathol Microbiol Scand $[B] 1976 ; 84,4: 225-8$.

${ }^{2}$ Spence L, Fauvel M, Bouchard S, Babiuk L, Saunders JR. Test for reovirus like agent. Lancet $1975 ; \mathrm{i}: 322$.

${ }^{3}$ Bryden AS, Davies Heather A, Thouless ME, Flewett TH. Diagnosis of rotavirus infection by cell culture. $J$ gen Microbiol 1977;10:121-5.

${ }^{4}$ Kalica AR, Purcell RH, Sereno MM, et al. A microtiter solid phase radioimmunoassay for detection of the human reo-virus like agent in stools. J Immunol 1977; 118, 4:1275-9.

${ }^{5}$ Scherrer R, Bernard S. Application of enzyme-linked immunosorbent assay (ELISA) to the detection of calf rotavirus and rotavirus antibodies. Ann Microbiol (Paris) $1977 ; 128,4 ; 499-510$.

- Yolken RH, Kim HW, Clem T, et al. Enzyme-linked immunosorbent assay (ELISA) for detection of human reovirus-like agent of infantile gastroenteritis. Lancet 1977;ii:263-6.

${ }^{7}$ Elliot KM. Acute diarrhoea in childhood. North Holland: Elsevier Excerpta Medica. Ciba Found Symp1976:1.

${ }^{8}$ Flewett TH, Bryden AS, Davies H. The viral flora of the faeces as seen by electron microscopy. J Clin Pathol 1974;27:603-8.

- Cohen J. Ribonucleic acid polymerase activity associated with purified calf rotavirus. J gen Virol 1977;36:395-402.

${ }^{10}$ Shirley Jane A, Beards GM, Thouless ME, Flewett TH. The influence of divalent cations on the stability of human rotavirus. Arch Virol 1981;67:1-9.

11 Wand antigenic relationships between viruses (rotaviruses) from acute gastroenteritis of children, calves, piglets, mice and foals. Infect Immun 1976;14:804-10.

12 Bridger JC. Location of type-specific antigens in calf rotaviruses. J Clin Microbiol 1978;8:625-8.

${ }^{13}$ Zissis GP, Lambert JP. Different serotypes of human rotavirus. Lancet 1978; 138 .

14 Thouless ME, Bryden AS, Flewett TH. Serotypes of human rotavirus. Lancet 1978 ; $\mathrm{i}: 39$.

15 Yolken RH, Wyatt RG, Zissis G, et al. Epidemiology of human rotavirus types 1 and 2 as studied by ELISA. $N$ Engl J Med 1978;299:1156-61.

${ }^{16}$ Beards GM, Pilfold JN, Thouless ME, Flewett TH. Rotavirus serotypes by serum neutralisation. J Med Virol 1980;5:231-7.

17 Caul EO, Ashley GR, Egglestone SI. An improved method for routine identification of faecal viruses using ammonium sulphate precipitation. FEMS Microbiol Lett 1978; 4:1-4.

18 House A. Economic impact of rotavirus and other neonatal disease agents of animals. J Am Vet Med Assoc 1978;173:573-6.

Requests for reprints to: Dr GM Beards, Regional Virus Laboratory, East Birmingham Hospital, Birmingham B9 5ST, England. 